Published in final edited form as:

Nat Neurosci. 2005 January ; 8(1): 10-12. doi:10.1038/nn0105-10.

\title{
Bridging the gap:
}

coupling single-cell oscillators in the suprachiasmatic nucleus

\author{
Christopher S Colwell \\ Department of Psychiatry and Biobehavioral Sciences at the University of California, Los Angeles, \\ 760 Westwood Plaza, Los Angeles, California 90024, USA. e-mail: ccolwell@mednet.ucla.edu
}

\begin{abstract}
Neurons in the mammalian master clock can maintain circadian rhythms in isolation, but must synchronize to function as a time-keeping system. A new study finds that gap junctions between neurons promote synchronous electrical activity and rhythmic behavior.
\end{abstract}

From daily sleep cycles to dinnertime, the circadian system is responsible for the timing of behavior and physiology. In mammals, the conductor of this multifaceted timing system can be localized to a pair of structures in the hypothalamus known as the suprachiasmatic nucleus $(\mathrm{SCN})^{1}$. Individual SCN neurons in isolation have the capacity to generate circadian oscillations in electrical activity, secretion and gene expression, but the cells drift out of phase with each other ${ }^{2}$. Understanding how individual oscillators remain synchronized in the intact SCN has been a fundamental gap in our knowledge of SCN function. In this issue, Long et al. 3 unambiguously demonstrate that SCN neurons are electrically coupled and that this coupling not only promotes synchronization of neural activity, but also is required for the maintenance of circadian rhythms in behavior.

The authors made intracellular recordings from pairs of neighboring SCN neurons. They found that about $25 \%$ of the neurons were electrically coupled and that these coupled cells showed synchronized spiking activity. The coupling strength and biophysical properties were similar to those measured in other types of coupled neurons ${ }^{4}$. Gap-junction channels are formed by a family of proteins called connexins. Connexin 36 (Cx36) is a major component of gap-junctionmediated electrical coupling in neurons ${ }^{4}$, and this seems to be the case in the SCN. Long et $a l$. found that the electrical coupling between SCN neurons was lost in Cx36 knockout mice $^{3}$. As compared to regions like the inferior olive, the new study found that the percentage of coupled cells in the SCN was relatively low ${ }^{3}$. This lower coupling frequency between SCN neurons seems to be consistent with our knowledge of SCN physiology. These clock cells do not show absolutely synchronized action potential generation; instead the population has coordinated firing rates that are high during the day and low during the night. However, it may be that some cell populations within the SCN are highly coupled and others not at all.

To determine whether gap-junction-mediated electrical coupling may also be involved in behavioral rhythmicity, the authors turned to the best-characterized behavioral output of the circadian system - namely, the wonderfully precise rhythms in wheel-running activity. In a light:dark cycle, both wild-type and $C x 36$ knockout mice synchronized to the lighting conditions and showed nocturnal activity rhythms characteristic of rodents. However, in a light:dark cycle, photic input organizes the temporal pattern of activity by synchronizing an endogenous clock to the period of the environmental signal (entrainment) as well as directly regulating activity (masking). To distinguish between these two effects of light, the authors placed the mice in constant darkness and measured their activity rhythms without light cues. In these conditions, the Cx36-deficient mice showed rhythms that were weaker and less coherent than those of controls. These deficits seemed to be due to a greater tendency for the 
$\mathrm{KO}$ mice to be active at inappropriate times in their daily cycle. The cycle-to-cycle variability in the onset of the daily activity bout was also higher in the mutant mice. Thus, without Cx36, the circadian clock still keeps time but lacks the temporal precision that typically characterizes the behavioral output.

The Long et $_{\text {al. }}{ }^{3}$ study helps to resolve a controversy about the presence and role of gap junctions in the SCN. The first suggestion that nonsynaptic mechanisms may link SCN neurons came from the observations that circadian rhythms in glucose utilization are present in the SCN before synapse formation 5 . In addition, when synaptic transmission is blocked by the removal of extracellular calcium, SCN neurons are still weakly coupled such that the activity of one cell increases the probability that a neighbor will generate an action potential ${ }^{6}$. A tracer (biocytin, neurobiotin or Lucifer yellow) placed in one SCN neuron spreads to clusters of surrounding cells ${ }^{7-9}$. Dye coupling definitively marks the presence of gap junctions. However, because the dye-coupled cells in these studies were not physiologically characterized, it was unclear whether they were neurons, astrocytes or other non-neuronal cell types.

Pharmacological gap junction blockers, such as halothane, disrupt circadian rhythms in SCN electrical activity and peptide secretion, as well as light-induced phase shifts of the circadian rhythm in wheel-running activity ${ }^{10}$. Unfortunately, these pharmacological tools are not very selective, and these agents have other effects besides blocking gap junctions. Anatomical studies have shown clear evidence for coupling between astrocytes and oligodendrocytes in the $\mathrm{SCN}^{11}$, but proof of neuron-to-neuron coupling has proven elusive until recently. First, results from freeze-fracture and immunocytochemistry provided evidence for $\mathrm{Cx} 36$-containing gap junctions between SCN neurons (Rash, J.E., et al., 749.11, Soc. Neurosci. Abstr., 2002). Now the new study ${ }^{3}$ demonstrates that SCN neurons are indeed electrically coupled and that this coupling is important for circadian rhythms in behavior (Fig. 1).

Like many good studies, this work raises as many questions as are answered by the experimental data. For example, we need to consider what signals are being spread from cell to cell via the gap junctions. Unlike chemical synapses, communication via gap junctions is bi-directional and allows passage of small molecules (up to $1 \mathrm{kDa}$ ), thus linking cells both electrically and metabolically. Signaling molecules such as cyclic AMP, cyclic GMP, $\mathrm{IP}_{3}$ and calcium may be able to pass between neurons through these connections. Future studies will have to consider the possibility that the passage of small molecules between cells may be as important as the direct passage of current. Gap-junction coupling also acts like an electrical filter in that some signals will pass more readily than others. During the day, SCN neurons undergo oscillations in membrane potential $(2-8 \mathrm{~Hz})$ that are driven by voltage-gated calcium currents, among other ionic mechanisms ${ }^{12}$. These slower changes in membrane potential should pass more effectively through gap junctions than the fast voltage changes that occur during an action potential.

One of the more tantalizing observations in the new study was the suggestion that the electrical coupling between SCN neurons may itself be subject to diurnal variation. The authors found that coupling was greater in the middle of the day, when rhythmic neural activity in the SCN peaks, than in the late day or early night. This observation is consistent with previous work demonstrating circadian variation in dye-coupling between SCN neurons ${ }^{8}$. In a few previous cases, changes in gap-junction permeability could be linked to changes in physiological function. For example, in the supraoptic nucleus of the hypothalamus, increased electrical coupling of oxytocin-secreting neurons may be a critical component of the milk-ejection reflex $^{13}$. These types of observations raise the possibility that gap junctions do not just allow the passive spread of current, but instead form an actively regulated communication system whose properties vary with the state of the organism. 
Another unresolved issue concerns the relative roles of electrical and chemical synaptic transmission in coupling SCN neurons. It is widely accepted that most SCN neurons express GABA and are likely to use this neurotransmitter for synaptic communication with other neurons in the SCN. In culture, GABA, acting through the $\mathrm{GABA}_{\mathrm{A}}$ receptor, can synchronize the electrical activity of SCN neurons 9,14 . Thus the synaptic release of GABA may act in concert with gap junctions to synchronize the neural activity of individual SCN oscillators. The SCN is made up of several cell populations whose specific functions we are just beginning to understand. One appealing hypothesis is that gap junctions may be more important for linking cells within a cell population, and that synaptic mechanisms may be more important for communication between SCN cell populations. Of course, it is also possible that SCN neurons are coupled by multiple, overlapping mechanisms, which may not be independent. Two studies looking at dye coupling within the $\mathrm{SCN}$ found that activation of $\mathrm{GABA}_{\mathrm{A}}$ receptors by muscimol actively inhibits the coupling 8,15 . Sorting out the relative role of these interacting coupling mechanisms should keep SCN watchers busy for years to come. These mice and this new research ${ }^{3}$ should help us bridge the gap between cellular coupling and circadian behavior.

\section{References}

1. Reppert SM, Weaver DR. Nature 2002;418:935-941. [PubMed: 12198538]

2. Welsh DK, Logothetis DE, Meisterm M, Reppert SM. Neuron 1995;14:697-706. [PubMed: 7718233]

3. Long MA, Jutras MJ, Connors BW, Burwell RD. Nat. Neurosci 2004;8:61-66. [PubMed: 15580271]

4. Connors BW, Long MA. Annu. Rev. Neurosci 2004;27:393-418. [PubMed: 15217338]

5. Reppert SM, Schwartz WJ. J. Neurosci 1984;4:1677-1682. [PubMed: 6737036]

6. Bouskila Y, Dudek FE. Proc. Natl. Acad. Sci. USA 1993;90:3207-3210. [PubMed: 8097315]

7. Jiang ZG, Yang YQ, Allen CN. Neuroscience 1997;77:1059-1066. [PubMed: 9130787]

8. Colwell CS. J. Neurobiol 2000;43:379-388. [PubMed: 10861563]

9. Shirakawa T, Honma S, Katsuno Y, Oguchi H, Honma KI. Eur. J. Neurosci 2000;12:2833-2838. [PubMed: 10971625]

10. Michel S, Colwell CS. Chronobiol. Int 2001;18:579-600. [PubMed: 11587083]

11. Rash JE, Yasumura T, Dudek FE, Nagy JI. J. Neurosci 2001;21:1983-2000. [PubMed: 11245683]

12. Pennartz CMA, de Jeu MTG, Bos NPA, Schaap J, Geurtsen AMS. Nature 2002;416:286-290. [PubMed: 11875398]

13. Hatton GI. Annu. Rev. Neurosci 1997;20:375-397. [PubMed: 9056719]

14. Liu C, Reppert SM. Neuron 2000;25:123-128. [PubMed: 10707977]

15. Shinohara K, Hiruma H, Funabashi T, Kimura F. Neuroscience 2000;96:591-596. [PubMed: 10717439] 

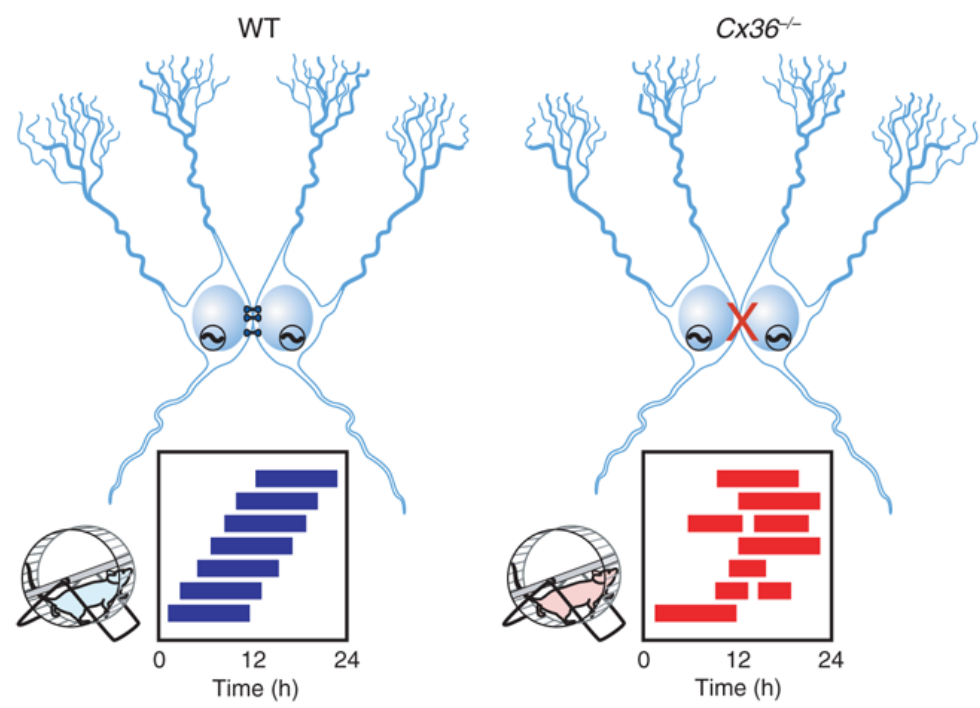

Figure 1.

Coupling of SCN neurons via gap junctions is important for the precision of circadian behavior. Top, schematic of pairs of SCN neurons (blue) from wild-type (WT) and Cx $36^{-/-}$mice. Individual SCN neurons contain the molecular machinery necessary to generate circadian oscillations. One gap in our knowledge is the lack of understanding of how these single-cell oscillators are coupled. The new study ${ }^{3}$ demonstrates that $\mathrm{SCN}$ neurons are coupled through direct electrical connections. This coupling is lost in mice deficient in Cx36. Bottom, schematics of wheel-running activity records from WT and Cx36-deficient mice. Animals maintained in constant darkness show rhythms driven by the endogenous timing system. Each horizontal row represents the activity record for a 24-hour day. Successive days are plotted from top to bottom. The colored bars represent activity. The WT mice express robust circadian rhythms of locomotor activity with period shorter then $24 \mathrm{~h}$. The onset of activity is typically under precise control. In contrast, the Cx36-deficient mice showed rhythms that were weaker and less coherent than controls. Without the $\mathrm{Cx} 36$, the circadian clock still keeps time but lacks the temporal precision that typically characterizes the behavioral output. 